\title{
Pair Production of Topological anti de Sitter Black Holes
}

\author{
R.B. Mann" \\ Dept. of Physics, University of Waterloo Waterloo, ONT N2L 3G1, Canada
}

PACS numbers: 04.70.Dy, 04.40.Nr, 04.60.-m

February 6, 2008

\begin{abstract}
The pair creation of black holes with event horizons of non-trivial topology is described. The spacetimes are all limiting cases of the cosmological $C$ metric. They are generalizations of the $(2+1)$ dimensional black hole and have asymptotically anti de Sitter behaviour. Domain walls instantons can mediate their pair creation for a wide range of mass and charge.
\end{abstract}

${ }^{1}$ email: mann@avatar.uwaterloo.ca 
Pair creation of black holes continues to afford us interesting insights into quantum gravity and the relationship between entropy and the number of quantum states of a black hole. It is a tunnelling process in which the mass-energy of the created pair of black holes is balanced by their negative potential energy in some background field, such as that of an electromagnetic field [1], a positive cosmological constant 22, a cosmic string [3] or a domain wall [4]. The amplitude for the process is approximated by $e^{-I_{i}}$, where $I_{i}$ is the action of the relevant instanton i.e. a Euclidean solution to the field equations which interpolates between the states before and after a pair of black holes is produced.

The $C$ metric solution of the Einstein-Maxwell equations may be interpreted as describing two oppositelycharged black holes undergoing uniform acceleration [5]. It contains conical singularities, which in general cannot be eliminated at both poles. These singularities are interpreted as representing "rods" or "strings" which provide the force necessary to accelerate the black holes. Removal of these singularities is generally obtained by adding additional forms of stress-energy which generate the background field required to provide the necessary accelerating force.

The purpose of this paper is to point out that the cosmological $C$-metrics [6] contain a rich array of Euclidean instantons that mediate pair production of black holes whose topology is of arbitrary genus. The genus zero solution corresponds to the set of Reissner-Nordström de Sitter instantons studied previously in the context of cosmological black hole pair production [2. The higher genus solutions are asymptotically anti-de Sitter, and correspond to instantons that are 4 dimensional generalizations of the 3 dimensional black hole [7]. Pair production of these black holes can take place in the presence of domain walls whose topology is the same as that of the produced black hole pairs.

The cosmological charged $C$-metric solution is [6]

$$
d s^{2}=\frac{1}{A^{2}(x-y)^{2}}\left[H(y) d t^{2}-H^{-1}(y) d y^{2}+G^{-1}(x) d x^{2}+G(x) d \varphi^{2}\right]
$$

where

$$
H(z)=a-b z^{2}-2 m A z^{3}-q^{2} A^{2} z^{4}=G(z)-\frac{k l^{2}}{A^{2}}
$$

$\Lambda=3 k / l^{2}$ being the cosmological constant and $A$ the acceleration parameter and $k= \pm 1$. The gauge field is

$$
F_{M}=-q d x \wedge d \varphi, \quad F_{E}=-q d t \wedge d y
$$

in the magnetic and electric cases respectively. Under the coordinate transformation $y=x-1 / A r, t=$ $A u-\int^{y} d z / H(z)$, the metric (11) may also be written as

$$
d s^{2}=H\left(x-\frac{1}{A r}\right) A^{2} r^{2} d u^{2}-2 d u d r-2 A r^{2} d u d x+r^{2}\left(G^{-1}(x) d x^{2}+G(x) d \varphi^{2}\right) \quad .
$$

The electric field becomes $F_{E}=-q d u \wedge\left(A d x+\frac{d r}{r^{2}}\right)$ and the magnetic field is unchanged.

The inner, outer and acceleration horizons are given by the first three roots of $H$ in ascending order respectively. The coordinate $r \in(0, \infty)$, whereas $x$ lies between either the smallest $\left(x_{1}, x_{2}\right)$ or largest $\left(x_{3}, x_{4}\right)$ pair of roots of $G$ so that the metric (4) has proper signature.

Removal of conical singularities in the $(x, \varphi)$ sector implies that

$$
G^{\prime}\left(x_{3}\right)=-G^{\prime}\left(x_{4}\right),
$$

with $\varphi$ periodically identified with period $\Delta \varphi=4 \pi /\left|G^{\prime}\left(x_{4}\right)\right|$. This condition can only be satisfied if $x_{3}=x_{4}$. A similar analysis for the smallest pair of roots implies that conical singularities can be removed if $x_{1}=x_{2}$. Conical singularities may also be avoided if $x_{2}=x_{3}$, in which case the point $x=x_{3}$ is an infinite proper distance from any allowed value of $x$.

Hence removal of conical singularities in the cosmological $C$ metric implies that $G(x)$ must have a double root. This apparently implies that the $(x, \varphi)$ sector shrinks to a point, but this is just a poor choice of coordinates. The proper distance between any adjacent pair $\left(x_{i}, x_{i+1}\right)$ of roots of $G$ is actually finite, as can be seen by setting $\varphi=\phi / \epsilon$ and and $x=\epsilon f(\lambda)$, where the roots $x_{i}=\epsilon f\left(\lambda_{i}\right)$ and $x_{i+1}=\epsilon f\left(\lambda_{i+1}\right)$ coincide as $\epsilon \rightarrow 0$. The parameters of $G$ must then be chosen so that it has a double root at $x=x_{i}=x_{i+1}$ as $\epsilon \rightarrow 0$. This constrains the acceleration parameter $A$ in terms of $m, q$ and $l$. 
For all possible pairs of degenerate roots the metric (㺼 becomes, as $\epsilon \rightarrow 0$,

$$
d s^{2}=-V(r) d T^{2}+\frac{d r^{2}}{V(r)}+r^{2}\left(d \lambda^{2}+s^{2}(\lambda) d \phi^{2}\right),
$$

where a coordinate transformation on $u$ has been performed,

$$
V(r)=-\frac{k}{l^{2}} r^{2}+b-\frac{2 m}{r}+\frac{q^{2}}{r^{2}}
$$

and $\phi$ has period $2 \pi$.

If the largest two roots of $H$ are degenerate then $b>0$, and the class of metrics obtained are of the Reissner-Nordström (anti)-de Sitter (RN(a)dS) type, of mass $m$ and charge $q$. However there is a surprise in that the parameter $b$ is completely arbitrary. A simple rescaling of parameters and coordinates allows $b$ to be set to unit magnitude without loss of generality if it is nonzero. If the middle two roots of $H$ are degenerate, then $b=-1$, and if the largest three roots are degenerate then $b=0$. There are therefore three possible forms for the function $s(\lambda)$ :

$$
\begin{array}{cl}
b=1, k= \pm 1 & s(\lambda)=\sin (\lambda) \\
b=0, k=-1 & s(\lambda)=1 \\
b=-1, k=-1 & s(\lambda)=\sinh (\lambda)
\end{array}
$$

It is easily checked that all of these spacetimes satisfy the Einstein-Maxwell equations with cosmological constant. The gauge field becomes

$$
F_{M}=q s(\lambda) d \lambda \wedge d \phi \quad F_{E}=-\frac{q}{r^{2}} d T \wedge d r
$$

in the magnetic and electric cases respectively.

The regularity requirements for the $C$ metric in the $b=1$ case for positive $\Lambda$ have been discussed previously [2]. The other spacetimes, however, have been overlooked in previous studies. The $b=1, \Lambda<0$ case is simply Reissner-Nordström anti de Sitter spacetime.

The two remaining spacetimes all have $\Lambda<0$ and $b<0$. Their $q=m=0$ versions were studied recently by Aminneborg et.al. 8 who showed that they can be understood as four-dimensional analogues of the three-dimensional black hole [7], by compactifying the $(\lambda, \phi)$ sector. For the metrics derived here this construction can also be carried out. For $b=1$ the topology of this sector is the 2 -sphere. For $b=0$, the coordinate $\lambda$ may be identified, and the $(\lambda, \phi)$ sector is a torus, whose unit area shall be chosen to be $4 \pi$ by identifying the lambda-coordinate with period 2 . For $b=-1$, the identifications may be carried out by mapping the $(\lambda, \phi)$ sector to the Poincaré disk under the transformation $\rho=\tanh (\lambda / 2)$, yielding

$$
d \lambda^{2}+s^{2}(\lambda) d \phi^{2}=\frac{1}{\left(1-\rho^{2}\right)^{2}}\left(d \rho^{2}+\rho^{2} d \phi^{2}\right)
$$

where $0 \leq \rho<1$. The Poincaré disk has an isomorphism group $\mathrm{SO}(2,1)$. Identifying points on the disk under any discrete subgroup of $\mathrm{SO}(2,1)$ yields a compact two-dimensional space of negative curvature, which necessarily has genus $g \geq 2$. The unit area of such surfaces is $4 \pi(g-1)$. These spaces may be constructed by symmetrically placing a polygon of $4 g$ sides at the center of the Poincare disk and identifying opposite sides. The edges of the polygon are geodesics of the Poincaré disk; these are circles whose extensions are orthogonal to the disk boundary. The simplest case is the octagon with $g=2$.

The $b \leq 0$ constructions hold for all values of $r$ and $T$ in (6). An analysis of the behaviour of $V(r)$ in (77) indicates that in this case it has at most two roots, corresponding to an inner and outer horizon, as with the usual RNadS metric. For $b=0$, there will be two horizons, provided

$$
27 l^{2} m^{4} \geq 16 Q^{6}
$$

with the extremal case saturating the inequality. For nonzero $b$ there will be event horizons provided

$$
m^{2} \leq \frac{l^{2}}{27} \frac{16-24 e^{2} b-16 b \sqrt{1-e^{2} b} e^{2}+6 b^{2} e^{4}+16 \sqrt{1-e^{2} b}}{e^{6}}
$$


where $e=\frac{2 \sqrt{2} q}{3 m}$. If $b=0$ (the genus 1 case) then the range of e is from 0 to 1 . Analysis of (14) in this case indicates that event horizons can (but need not) exist provided $q<m$. If $b=-1$ then there is no (obvious) upper limit on e, and event horizons can exist for arbitrarily large values of $q$ relative to $m$.

The topology of the outer event horizon is $H_{g}^{2}$, where $H_{g}^{2}$ is a two-dimensional surface of genus $g$. The entire spacetime has topology $R^{2} \times H_{g}^{2}$. An analysis of the quasilocal mass 11 and charge contained within a surface of topology $H_{g}$ at a fixed value of the coordinate $r$ centered about the origin indicates, in the limit of large $r$, that $q\left(|g-1|+\delta_{g, 1}\right)$ and $m\left(|g-1|+\delta_{g, 1}\right)$ are the conserved charge and mass parameters of the black hole.

Pair production of these black holes may be achieved using the domain wall construction of ref. [4]. The topology of the Riemannian section is $R^{2} \times H_{g}^{2}$, where the $R^{2}$ factor is like a bell. Two copies of this manifold may be matched together at a radius $r$ determined by the matching condition [9, 10]

$$
\sqrt{V(r)-\dot{r}^{2}}=2 \pi \sigma
$$

where $\sigma$ is the energy per unit area of the domain wall, whose topology is $S^{1} \times H_{g}^{2}$, and the overdot refers to the derivative with respect to Euclidean proper time. The Riemannian section is two bells glued together along their open ends at a ridge; it has topology $S^{2} \times H_{g}^{2}$ and corresponds to static a domain wall configuration with two surfaces at which the Killing field $\frac{d}{d \tau}$ vanishes, where $\tau$ is the Euclidean time parameter.

Equation (15) may be interpreted as the equation describing the motion of a fictitious particle in a potential $v=V-(2 \pi \sigma r)^{2}$. Static solutions, which have energy zero, may be obtained by solving (15) under the condition $\partial v / \partial r=0$. Non-static solutions are obtained by matching the period of the RNadS black hole with an integer multiple of

$$
\beta_{w}=\oint_{r \min }^{r \max } d \tau=\oint_{r \min }^{r \max } \frac{d r}{\sqrt{V\left(V-(2 \pi \sigma r)^{2}\right)}}
$$

which is the period of the domain wall evolution between the extrema rmin and rmax where $\dot{r}$ vanishes.

For simplicity, I shall consider static solutions; details including non-static solutions shall appear in a forthcoming paper 12]. These occur when

$$
r_{s}=\frac{3 m b+\sqrt{1-8 e^{2} b}}{2}
$$

if $b \neq 0$ and at $r_{s}=\frac{2 q^{2}}{3 m}$ if $b=0$. Note that for $b<0$ there is no solution with zero charge. The squared mass of the created black holes is

$$
m^{2}=\frac{1}{54}\left[36 q^{2}+\frac{b}{4 \pi^{2} \sigma^{2} l^{2}-1}\left(l^{3}+\sqrt{\left(l^{2}+12 q^{2}\left(1-4 \pi^{2} \sigma^{2} l^{2}\right)^{3}\right.}\right)\right]
$$

for nonzero $b$ and by

$$
m^{2}=\frac{4 q^{3}}{3 \sqrt{3} l} \sqrt{4 \pi^{2} \sigma^{2} l^{2}-1}
$$

if $b=0$.

The Euclidean action for these instantons is

$$
I=\int d^{4} x \sqrt{g}\left(-\frac{R}{16 \pi}+\frac{F^{2}}{16 \pi}+\mathcal{L}_{c}+\mathcal{L}_{d}\right)
$$

where $\mathcal{L}_{c}$ is the cosmological Lagrangian and $\mathcal{L}_{d}$ the domain-wall Lagrangian. The former may be taken to be that of the squared field strength of a 3 -form or simply the constant $3 \frac{k}{8 \pi l^{2}}$. The domain wall Lagrangian can be that of a membrane current coupling to the 3-form [10] or that of a scalar field $\Phi$ whose potential $\mathcal{V}(\Phi)$ is everywhere positive [ 4 (and so its Euclidean action is always negative). There are no boundary terms because the instantons considered here are compact and without boundary.

Using the Einstein field equations (20) becomes

$$
I=\int d^{4} x \sqrt{g}\left(-3 \frac{k}{8 \pi l^{2}}+\frac{F^{2}}{16 \pi}-\mathcal{V}(\Phi)\right)
$$


which yields in, say, the $g=2$ case

$$
P=\exp \left[\left(2 \pi \sigma r_{s}^{2} \sqrt{V\left(r_{s}\right)} \beta-\frac{q^{2}}{r_{s} r_{+}}\left(r_{s}-r_{+}\right) \beta+\frac{k\left(r_{+}^{3}-r_{s}^{3}\right) \beta}{l^{2}}-\frac{1}{8 \pi \sigma^{2}}\right)\right]
$$

for the probability of pair creation of the black holes in the magnetic case; the electric case is similar but entails the incorporation of an additional surface term that vanishes in the magnetic case [2]. Here $r_{+}$is the location of the outer horizon and $\beta$ the instanton period for the RNadS black hole. The above expression includes the case $k=1(\mathrm{RNdS})$; if the $\sigma$-dependent terms are omitted, and $r_{s}$ is taken to be the location of the cosmological horizon, then the results of ref. [2] are recovered. The probability (22) is relative to that for creation of a domain wall with no black holes or relativisitic 3 -form.

To summarize, the C-metric has been shown to reduce to a set of metrics whose Euclidean sections can be interpreted as instantons corresponding to the pair creation of black holes with event horizons of arbitrary topology. The Lorentzian sections are spacetimes that are asymptotically anti de Sitter and which can be interpreted as generalizations of the $(2+1)$ dimensional black hole. A more detailed study of these black holes and their pair creation will appear in a forthcoming paper 12 .

\section{Acknowledgements}

This work was supported by the Natural Sciences and Engineering Research Council of Canada. I am grateful to J. Creighton for interesting discussions on this subject.

\section{References}

[1] H.F. Dowker, J.P. Gauntlett, D.A. Kastor and J. Traschen, Phys. Rev. D 49, 2909 (1994); H.F. Dowker, J.P. Gauntlett, S.B. Giddings and G.T. Horowitz, Phys. Rev. D 50, 2662 (1994); S.W. Hawking, G.T. Horowitz and S.F. Ross, Phys. Rev. D51, 4302 (1995).

[2] R.B. Mann and S.F. Ross, Phys. Rev. D52, 2254 (1995).

[3] S.W. Hawking and Simon F. Ross, Phys. Rev. Lett. 75 (1995) 3382; R. Emparan Phys. Rev. Lett. 75 (1995) 3386 .

[4] R.R. Caldwell, G.W. Gibbons, and A. Chamblin, "Pair Creation of Black Holes by Domain Walls", hep-th/9602216.

[5] W. Kinnersley and M. Walker, Phys. Rev. D 2, 1359 (1970).

[6] J.F. Plebanski and M. Demianski, Ann. Phys. 98, 98 (1976).

[7] M. Banados, C. Teitelboim and J. Zanelli, Phys. Rev. Lett. 69, (1992) 1849; M. Banados, M. Henneaux, C. Teitelboim and J. Zanelli, Phys. Rev. D 48, (1993) 1506.

[8] S. Aminneborg, I Bengtsson, S. Holst and P. Peldan, "Making Anti de Sitter Black Holes" gr-qc/9604022.

[9] W.A. Hiscock Phys. Rev. D35, (1987) 1161.

[10] A. Aurelia, R. Kissack, R.B. Mann and M. Spallucci, Phys. Rev. D35 (1987) 2961.

[11] J.D. Brown and J.W. York, Phys. Rev. D 47, 1420 (1993); J.D. Brown, J. Creighton and R.B. Mann, Phys. Rev. D 506394 (1994).

[12] R.B. Mann, in preparation. 